(C) 2018 IEEE. Personal use of this material is permitted. Permission from IEEE must be obtained for all other uses, in any current or future media, including reprinting/republishing this material for advertising or promotional purposes, creating new collective works, for resale or redistribution to servers or lists, or reuse of any copyrighted component of this work in other works. 


\title{
Codebook Based Minimum Subspace Distortion Hybrid Precoding for Millimeter Wave Systems
}

\author{
Zhitong $\mathrm{Ni}^{1}$, J. Andrew Zhang ${ }^{2}$, Kai Yang ${ }^{1}$, Fei Gao ${ }^{1}$ and Zhen $\mathrm{Gao}^{1}$ \\ ${ }^{1}$ Beijing Institute of Technology, School of Information and Electronics, China \\ ${ }^{2}$ University of Technology Sydney, Global Big Data Technologies Centre (GBDTC), Australia \\ zhitongni@bit.edu.cn; Andrew.Zhang@uts.edu.au; yangkai@ bit.edu.cn.
}

\begin{abstract}
Hybrid precoding is adopted for millimeter wave (mmWave) communications to offer a good trade-off between hardware complexity and system performance. In this paper, we investigate a codebook based hybrid precoder for single-user mmWave systems with large antenna arrays. We exploit the sparse nature of mmWave channels to transform the hybrid precoding design problem into a vector space distortion optimization problem which is only related to the radio frequency (RF) precoder. A near optimal solution for the RF optimization problem is derived with the assumption of the perfect channel state information (CSI) at the transmitter, which is practically very difficult to obtain. To reduce the requirement of the CSI at the transmitter, we propose the codebook based minimum subspace distortion (MSD) hybrid precoding algorithm, which obtains CSI at the combiner side and returns the index of optimal RF codewords and the baseband precoder through a limited feedback channel. Simulation results are provided and validate the effectiveness of our proposed hybrid precoding algorithm.
\end{abstract}

\section{INTRODUCTION}

Hybrid array is a promising technique that provides a flexible compromise between system performance and hardware cost for millimetre wave (mmWave) communications $[1,2]$. It transmits parallel data streams but adopts less number of RF chains compared with traditional fully digital solutions. Due to the special combined digital and analog array structure [3], hybrid precoding at the transmitter and hybrid combining at the receiver are challenging design problems.

Most prior work on hybrid precoding design focused on improving the spectral efficiency with the assumption of perfect channel state information at the transmitter (CSIT) [4-7]. In [4], hybrid precoding algorithms were designed to formulate the precoding/combining problem as a sparse reconstruction problem. Using the principle of basis pursuit, their proposed hybrid precoders approach to the optimal unconstrained precoders with the perfect CSIT. Following [4], a substantial number of papers investigated the hybrid precoder designs, aiming to approaching the performance of a fully digital array under various situations. The authors in [5] proposed the hybrid precoding algorithm for wideband systems and maximized the sum rate over different subcarriers with a MIMO-OFDM architecture. In [6], a RF precoding algorithm with the assumption of perfect CSIT was proposed for multi-user mmWave systems by matching the phase of different users' channels. In [7], the authors proposed low complexity hybrid precoding algorithms for the narrow band single-user mmWave system and maximized either diversity or spatial multiplexing gain also with the assumption of perfect CSIT. In practice, the perfect CSIT is difficult to obtain in mmWave systems, especially when the number of antennas is large. Therefore, it is important to propose new hybrid precoding algorithms without the assumption of perfect CSIT.

Codebook based hybrid precoding can potentially reduce the requirement of channel knowledge. In [8], utilizing the sparse nature of mmWave channels, the authors investigated mmWave channel estimation and proposed a completed hybrid precoder design. The hybrid precoder developed based on the estimated channel achieves performance close to that of digital solutions. The authors in [9] proposed a codebookbased hybrid precoding algorithm with CSIT obtained via a beam sweep procedure. The developed solutions in [9], however, have a high computational complexity and is designed only for the system with a single receiver antenna. In [10], the optimal baseband precoders based on RF codebook were derived for OFDM-based hybrid precoding systems. However, the computational complexity is quite high, and the channel correlation was not exploited to improve the performance and reduce the complexity. The work in [11] leveraged the sparsity of mmWave channel and adopted a quantized codebook to obtain the hybrid precoder through a limited feedback channel. It performs well when the number of scatters in the channel is small, but its performance degrades significantly in a multi-path channel. In [12], a codebook-based hybrid precoding design was studied for the subarray architecture. The complexity of the scheme is reduced at the cost of performance degradation.

In this paper, we develop a codebook-based min- 
imum subspace distortion (MSD) hybrid precoding algorithm for narrow-band single-user mmWave MIMO systems. We analyze the hybrid precoding optimization problem and define the metric of vector space distortion between RF precoder and the optimal fully digital precoder. Then, we derive a near optimal solution for the hybrid precoder by minimizing the space distortion between the RF precoder and the optimal fully digital precoder. Considering the difficulty of obtaining CSIT, we propose a codebook based RF precoder consisting of limited number of codewords, and transform the subspace distortion optimization problem into a codeword selection problem. Finally, we optimize the codebook based RF precoder by minimizing the subspace distortion and return the index of optimal codewords and the optimal baseband precoder to the transmitter, such that the CSIT is not required.

The notations are used throughout this paper: a denotes a vector, A denotes a matrix, italic English letters like $N$ and lower-case Greek letters $\alpha$ are a scalar, $\angle a$ is the phase angle of complex value $a$. $|\mathbf{A}|, \mathbf{A}^{T}, \mathbf{A}^{H}, \mathbf{A}^{\dagger}$ represent determinant value, transpose, conjugate transpose, pseudo inverse respectively. We denote Frobenius norm of a matrix as $\|\mathbf{A}\|_{F}$, and p-norm of a vector as $\|\mathbf{a}\|_{p}$. We use $\otimes$ as Kronecker product. $\operatorname{diag}\left(\alpha_{1}, \cdots, \alpha_{k}\right)$ is a diagonal matrix whose diagonal elements are $\alpha_{1}, \cdots, \alpha_{k} .[\mathbf{A}]_{N}$ is the $N^{t h}$ column of a matrix. $\mathbb{E}(\cdot)$ is the expectation of a random variable.

\section{System ModeL}

We consider the downlink of single-user mmWave MIMO system as shown in Fig. 1, where the transmitter adopts a hybrid array with $N_{\mathrm{Tx}}$ antennas and $N_{\mathrm{P}}$ RF chains, sending $N_{\mathrm{S}} \leq N_{\mathrm{P}}$ data streams to the receiver. The receiver adopts a fully digital array with $N_{\mathrm{Rx}}$ antennas. The downlink mmWave channel is not necessary to be symmetric with the uplink channel. The number of antennas is set to be large to achieve high array gain but it is impractical to install an RF chain for each antenna. As such, we assume $N_{\mathrm{P}} \ll N_{\mathrm{Tx}}$. The transmitted signal is given by

$$
\mathbf{x}=\mathbf{F}_{\mathrm{RF}} \mathbf{F}_{\mathrm{BB}} \mathbf{s}
$$

where $\mathbf{s}$ is an $N_{\mathrm{S}} \times 1$ data stream vector, which is precoded by an $N_{\mathrm{P}} \times N_{\mathrm{S}}$ baseband precoder $\mathbf{F}_{\mathrm{BB}}$ in the digital (baseband) domain. After the baseband precoding, DACs and RF chains are used to convert the digital signal to the analog (RF) domain, and the analog signal is precoded by an $N_{\mathrm{Tx}} \times N_{\mathrm{P}} \mathrm{RF}$ precoder $\mathbf{F}_{\mathrm{RF}}$, which is implemented by analog phase shifters.

The entries of $\mathbf{F}_{\mathrm{RF}}$ have a constant modulus with $N$ phase shifting values, i.e.,

$\left[\mathbf{F}_{\mathrm{RF}}\right]_{m, n}=\frac{1}{\sqrt{N_{\mathrm{Tx}}}} e^{j \varphi_{m, n}}, \varphi_{m, n} \in\left\{\frac{2 \pi}{N}, \frac{4 \pi}{N}, \cdots, 2 \pi\right\}$,

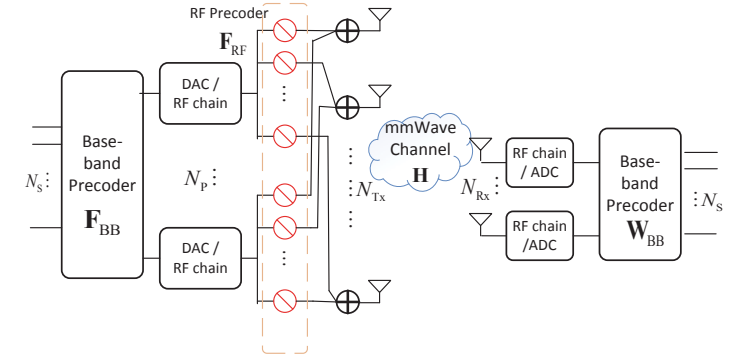

Fig. 1. Illustration of the single-user mmWave MIMO system, where the transmitter uses a hybrid array and the receiver uses a fully digital array.

where $\varphi_{m, n}$ represents the angle of the phase shifter. Considering the codebook-based hybrid precoder design, we assume that each column of $\mathbf{F}_{\mathrm{RF}}$ is specified with an RF codeword, i.e.,

$$
\mathbf{F}_{\mathrm{RF}}=\left[\mathbf{f}_{i_{1}}, \mathbf{f}_{i_{2}}, \cdots, \mathbf{f}_{i_{N_{\mathrm{P}}}}\right], \mathbf{f}_{i} \in \mathcal{C}_{F},
$$

where $\mathcal{C}_{F}$ is the RF codebook. Due to the total power constraints, we normalize the hybrid precoder, such that $\left\|\mathbf{F}_{\mathrm{RF}} \mathbf{F}_{\mathrm{BB}}\right\|_{F}^{2}=N_{\mathrm{S}}$

At the receiver, the received signal is transformed to digital signal via $N_{\mathrm{Rx}}$ RF chains and ADCs. Then, an $N_{\mathrm{Rx}} \times N_{\mathrm{S}}$ digital combiner $\mathbf{W}_{\mathrm{BB}}$ is adopted to process the digital signal,

$$
\mathbf{y}=\mathbf{W}_{\mathrm{BB}}^{H}\left(\mathbf{H F}_{\mathrm{RF}} \mathbf{F}_{\mathrm{BB}} \mathbf{s}+\mathbf{n}\right),
$$

where $\mathbf{H}$ with the dimension of $N_{\mathrm{Rx}} \times N_{\mathrm{Tx}}$ represents the narrow-band mmWave channel model, and $\mathbf{n} \sim \mathcal{C N}\left(\mathbf{0}, \sigma^{2} \mathbf{I}\right)$ denotes the complex Gaussian noise.

MmWave channels are highly directional and have limited scattering [4]. We consider the downlink channel with a beam-space geometric model. The channel model is not necessary to be symmetric with the uplink channel model and is given by

$$
\mathbf{H}=\rho \sum_{c=1}^{L_{\mathrm{C}}} \sum_{l=1}^{L_{\mathrm{R}}} \alpha_{c, l} \mathbf{e}_{\mathrm{R}}\left(\theta_{c, l}^{r}, \varphi_{c, l}^{r}\right) \mathbf{e}_{\mathrm{T}}^{H}\left(\theta_{c, l}^{t}, \varphi_{c, l}^{t}\right),
$$

where $\rho=\sqrt{\frac{N_{\mathrm{Tx}} N_{\mathrm{Rx}}}{L_{\mathrm{R}} L_{\mathrm{C}}}}$ is the normalized factor, $L_{\mathrm{C}}$ is the number of clusters and each cluster contributes $L_{\mathrm{R}}$ propagation paths between the transmitter and the receiver, $\alpha_{c, l}$ is the channel complex gain, $\theta_{c, l}^{t}$ and $\varphi_{c, l}^{t}$ are the elevation and azimuth angles of departure (AoD), $\theta_{c, l}^{r}$ and $\varphi_{c, l}^{r}$ are elevation and azimuth angles of arrival (AoA), $\mathbf{e}_{\mathrm{R}}\left(\theta_{c, l}^{r}, \varphi_{c, l}^{r}\right)$ and $\mathbf{e}_{\mathrm{T}}\left(\theta_{c, l}^{t}, \varphi_{c, l}^{t}\right)$ are the received and the transmitted array response vectors. For a uniform linear array (ULA), the array response vector at the transmitter is given by

$$
\mathbf{e}_{\mathrm{T}}(\theta)=\frac{1}{\sqrt{N_{\mathrm{Tx}}}} \exp \left[\frac{j 2 \pi}{\lambda} d \sin \theta\left(0, \cdots, N_{\mathrm{Tx}}-1\right)\right]^{T},
$$

where $\lambda$ is the wave length and $d$ is the inter-element 
spacing. Note that $\varphi$ is not included in (6) as the response vector is invariant in the azimuth domain.

\section{Problem Formulation}

The goal of this paper is to design $\mathbf{F}_{\mathrm{RF}}, \mathbf{F}_{\mathrm{BB}}$ and $\mathbf{W}_{\mathrm{BB}}$ such that the spectral efficiency (SE) is maximized. The optimization problem is given by

$$
\begin{aligned}
& \left\{\mathbf{F}_{\mathrm{RF}}^{\star}, \mathbf{F}_{\mathrm{BB}}^{\star}, \mathbf{W}_{\mathrm{BB}}^{\star}\right\} \\
& =\arg \max \log _{2} \mid \mathbf{I}+\frac{S N R}{N_{\mathrm{S}}} \mathbf{W}_{\mathrm{BB}}^{H} \mathbf{H F}_{\mathrm{RF}} \mathbf{F}_{\mathrm{BB}} \mathbf{F}_{\mathrm{BB}}^{H} \times \\
& \mathbf{F}_{\mathrm{RF}}^{H} \mathbf{H} \mathbf{W}_{\mathrm{BB}} \\
& \text { s.t. }\left\|\mathbf{F}_{\mathrm{RF}} \mathbf{F}_{\mathrm{BB}}\right\|_{F}^{2}=N_{\mathrm{S}},\left\|\mathbf{W}_{\mathrm{BB}}\right\|_{F}^{2}=N_{\mathrm{S}} \text {, } \\
& \mathbf{F}_{\mathrm{RF}}=\left[\mathbf{f}_{i_{1}}, \mathbf{f}_{i_{2}}, \cdots, \mathbf{f}_{i_{N_{\mathrm{P}}}}\right] \text {, } \\
& \mathbf{f}_{i} \in \mathcal{C}_{F}, i \in\left\{1,2, \cdots, N_{\mathrm{CB}}\right\},
\end{aligned}
$$

where $\mathbf{f}_{i}$ is the $i^{\text {th }}$ codeword in the $p$-bit quantized codebook $\mathcal{C}_{F}$, of which size is $N_{\mathrm{CB}}$, i.e., $N_{\mathrm{CB}}=2^{p}$.

The formulated problem above is difficult to solve due to several constraints, including the coupling between $\mathbf{W}_{\mathrm{BB}}, \mathbf{F}_{\mathrm{RF}}$ and $\mathbf{F}_{\mathrm{BB}}$, the constant-modulus requirement of $\mathbf{F}_{\mathrm{RF}}$, and the non-convex nature of the SE. Another practical difficulty is to know CSIT, which requires the receiver to estimate a large dimensional channel matrix and feedback it to the transmitter. In the following, we will address these difficulties and simplify the hybrid precoding design problem.

We consider to decouple the hybrid precoder and the digital combiner by separating the precoder design from the combiner design, which is equivalent to maximizing the mutual information at transmitter and receiver, respectively [4]. At the precoder side, the mutual information at the transmitter is given by

$$
\begin{aligned}
I_{t} & =\log _{2}\left|\mathbf{I}+\frac{S N R}{N_{\mathrm{S}}} \mathbf{H} \mathbf{F}_{\mathrm{RF}} \mathbf{F}_{\mathrm{BB}} \mathbf{F}_{\mathrm{BB}}^{H} \mathbf{F}_{\mathrm{RF}}^{H} \mathbf{H}^{H}\right| \\
& =\log _{2}\left|\mathbf{I}+\frac{S N R}{N_{\mathrm{S}}} \boldsymbol{\Sigma}^{2} \mathbf{V}^{H} \mathbf{F}_{\mathrm{RF}} \mathbf{F}_{\mathrm{BB}} \mathbf{F}_{\mathrm{BB}}^{H} \mathbf{F}_{\mathrm{RF}}^{H} \mathbf{V}\right|,
\end{aligned}
$$

where $\mathbf{H}=\mathbf{U} \boldsymbol{\Sigma} \mathbf{V}^{H}$ is the singular value decomposition (SVD) of the channel.

Considering the sparse nature of mmWave channels, the mutual information at the transmitter can be simplified by separating the channel singular matrices into two partitions [10], i.e.,

$$
\mathbf{U}=\left[\overline{\mathbf{U}}, \mathbf{U}_{e x}\right], \boldsymbol{\Sigma}=\operatorname{diag}\left(\overline{\boldsymbol{\Sigma}}, \boldsymbol{\Sigma}_{e x}\right), \mathbf{V}=\left[\overline{\mathbf{V}}, \mathbf{V}_{e x}\right],
$$

where $\bar{\Sigma}$ is the first $N_{\mathrm{S}}$ entries of the diagonal matrix $\Sigma$ with dimension of $N_{\mathrm{S}} \times N_{\mathrm{S}}, \overline{\mathbf{U}}$ and $\overline{\mathbf{V}}$ are the first $N_{\mathrm{S}}$ columns of $\mathbf{U}$ and $\overline{\mathbf{V}}$, respectively. The matrix $\boldsymbol{\Sigma}_{e x}$ is nearly a zero matrix due to the sparsity of the mmWave channel when $N_{s}$ is not very small. Taking the same steps to that in (12)-(15) of [4], $I_{t}$ is approximately written as

$I_{t} \approx \log _{2}\left(\left|\mathbf{I}+\frac{S N R}{N_{\mathrm{S}}} \overline{\mathbf{\Sigma}}^{2}\right|\right)-\left(N_{\mathrm{S}}-\left\|\overline{\mathbf{V}}^{H} \mathbf{F}_{\mathrm{RF}} \mathbf{F}_{\mathrm{BB}}\right\|_{F}\right)$,

where the second term in (10) is known as the chordal distance between $\mathbf{F}_{\mathrm{RF}} \mathbf{F}_{\mathrm{BB}}$ and $\overline{\mathbf{V}}$ on the Grassmann manifold [13]. Hence, maximizing $I_{t}$ is equivalent to minimizing the chordal distance. We adopt the assumption proposed in [4], that the hybrid precoder is supposed to be made close to the eigenspace of the dominant channel, to replace the chordal distance with the Euclidean distance that equals $\left\|\mathbf{F}_{\mathrm{RF}} \mathbf{F}_{\mathrm{BB}}-\overline{\mathbf{V}}\right\|_{F}$. Hence, the optimization problem is transformed into

$$
\begin{aligned}
& \left\{\mathbf{F}_{\mathrm{RF}}^{\star}, \mathbf{F}_{\mathrm{BB}}^{\star}\right\}=\arg \min \left\|\mathbf{F}_{\mathrm{RF}} \mathbf{F}_{\mathrm{BB}}-\overline{\mathbf{V}}\right\|_{F} \\
& \text { s.t. }\left\|\mathbf{F}_{\mathrm{RF}} \mathbf{F}_{\mathrm{BB}}\right\|_{F}^{2}=N_{\mathrm{S}} \text {, } \\
& \mathbf{F}_{\mathrm{RF}}=\left[\mathbf{f}_{i_{1}}, \mathbf{f}_{i_{2}}, \cdots, \mathbf{f}_{i_{N_{\mathrm{P}}}}\right] \text {, } \\
& \mathbf{f}_{i} \in \mathcal{C}_{F}, i \in\left\{1,2, \cdots, N_{\mathrm{CB}}\right\} \text {. }
\end{aligned}
$$

The coupling between $\mathbf{F}_{\mathrm{RF}}$ and $\mathbf{F}_{\mathrm{BB}}$ can be addressed by using the Least Square (LS) method, which minimizes the residual error between columns of $\mathbf{F}_{\mathrm{RF}} \mathbf{F}_{\mathrm{BB}}$ and those of $\overline{\mathbf{V}}$. Given an RF precoder, the optimal baseband precoder is given by

$$
\mathbf{F}_{\mathrm{BB}}^{\star}=\mathbf{F}_{\mathrm{RF}}^{\dagger} \overline{\mathbf{V}} \text {. }
$$

Substituting (12) into the objective function of (11), we define the vector space distortion between $\mathbf{F}_{\mathrm{RF}}$ and $\overline{\mathbf{V}}$ as

$$
\begin{aligned}
d\left(\mathbf{F}_{\mathrm{RF}}, \overline{\mathbf{V}}\right) & =\left\|\mathbf{F}_{\mathrm{RF}} \mathbf{F}_{\mathrm{BB}}-\overline{\mathbf{V}}\right\|_{F} \\
& =\left(\sum_{i}^{N_{\mathrm{S}}}\left\|\mathbf{F}_{\mathrm{RF}} \mathbf{F}_{\mathrm{RF}}^{\dagger} \overline{\mathbf{v}}_{i}-\overline{\mathbf{v}}_{i}\right\|_{2}^{2}\right)^{\frac{1}{2}} \\
& =\left(\sum_{i}\left\|\left(\mathbf{F}_{\mathrm{RF}} \mathbf{F}_{\mathrm{RF}}^{\dagger}-\mathbf{I}_{N_{\mathrm{Tx}}}\right) \overline{\mathbf{v}}_{i}\right\|_{2}^{2}\right)^{\frac{1}{2}} \\
& =\left\|\left(\mathbf{F}_{\mathrm{RF}} \mathbf{F}_{\mathrm{RF}}^{\dagger}-\mathbf{I}_{N_{\mathrm{Tx}}}\right) \overline{\mathbf{V}}\right\|_{F} .
\end{aligned}
$$

We note that $d\left(\mathbf{F}_{\mathrm{RF}}, \overline{\mathbf{V}}\right)$ equals zero when $\mathbf{F}_{\mathrm{RF}}$ is invertible. However, due to the constraints on the number of RF chains, the RF precoder makes $d\left(\mathbf{F}_{\mathrm{RF}}, \overline{\mathbf{V}}\right)$ larger than zero. To minimize the error of subspace distortion, the optimal RF precoder is given by

$$
\begin{aligned}
\mathbf{F}_{\mathrm{RF}}^{\star}=\arg \min & \left\|\left(\mathbf{F}_{\mathrm{RF}} \mathbf{F}_{\mathrm{RF}}^{\dagger}-\mathbf{I}_{N_{\mathrm{Tx}}}\right) \overline{\mathbf{V}}\right\|_{F} \\
\text { s.t. } & \mathbf{F}_{\mathrm{RF}}=\left[\mathbf{f}_{i_{1}}, \mathbf{f}_{i_{2}}, \cdots, \mathbf{f}_{\left.i_{N_{\mathrm{P}}}\right],}\right. \\
& \mathbf{f}_{i} \in \mathcal{C}_{F}, i \in\left\{1,2, \cdots, N_{\mathrm{CB}}\right\} .
\end{aligned}
$$

The solution to the combiner design is either to maximize the spectral efficiency or to minimize the mean squared error between the data streams and the received signal [7]. For simplicity, we adopt the former 
one where the optimum combiner $\mathbf{W}_{\mathrm{BB}}^{\star}$ is given by

$$
\mathbf{W}_{\mathrm{BB}}^{\star}=\overline{\mathbf{U}} \text {. }
$$

\section{Hybrid Precoder Design with Perfect CSIT}

In this section, we derive the optimal RF precoder that solves the problem in (14) with the assumption of perfect CSIT. It can be derived that the optimal RF precoder without constraints is given by

$$
\mathbf{F}_{\mathrm{RF}}=[\mathbf{V}]_{1: N_{\mathrm{P}}} \mathbf{A},
$$

where $\mathbf{A}$ is an $N_{\mathrm{P}} \times N_{\mathrm{P}}$ full rank matrix. To verify, we substitute $\mathbf{F}_{\mathrm{RF}}$ in (16) to (14) and obtain

$$
\begin{aligned}
& \left\|\left(\mathbf{F}_{\mathrm{RF}} \mathbf{F}_{\mathrm{RF}}^{\dagger}-\mathbf{I}_{N_{\mathrm{Tx}}}\right) \overline{\mathbf{V}}\right\|_{F} \\
= & \left\|\left([\mathbf{V}]_{1: N_{\mathrm{P}}} \mathbf{A A}^{-1}[\mathbf{V}]_{1: N_{\mathrm{P}}}^{H}-\mathbf{I}_{N_{\mathrm{Tx}}}\right) \overline{\mathbf{V}}\right\|_{F} \\
= & \left\|[\mathbf{V}]_{1: N_{\mathrm{P}}}[\mathbf{V}]_{1: N_{\mathrm{P}}}^{H} \overline{\mathbf{V}}-\overline{\mathbf{V}}\right\|_{F} \\
= & \|\overline{\mathbf{V}}-\overline{\mathbf{V}}\|_{F}=0 .
\end{aligned}
$$

To account for the modulus constraint of phase shifters, the RF precoder matrix is converted to $\mathbf{F}_{\mathrm{RF}}=$ $\exp \left([\mathbf{V}]_{1: N_{\mathrm{P}}} \mathbf{A}\right)$. Therefore, the practical RF precoder with phase shifter constraints results in normalization error, which is defined as

$$
e_{\mathrm{n}}=\left\|[\mathbf{V}]_{1: N_{\mathrm{P}}} \mathbf{A}-\exp \left([\mathbf{V}]_{1: N_{\mathrm{P}}} \mathbf{A}\right)\right\|_{F} .
$$

We note that the matrix $\mathbf{A}$ is seen as a linear transformation matrix, which changes the norm of each vectors in $[\mathbf{V}]_{1: N_{\mathrm{P}}}$. Hence, we need to design $\mathbf{A}$ to minimize $e_{\mathrm{n}}$. Consider the design of the optimal unconstrained RF precoder in (16), which is related to the channel singular vectors. These singular vectors take a DFT structure for uniform arrays as $N \rightarrow \infty$ [4]. Hence a reasonable substitution for the global optimal solution of $\mathbf{A}$ is given by

$$
\mathbf{A}^{\star}=\exp \left(j \angle[\mathbf{V}]_{1: N_{\mathrm{P}}}\right)[\mathbf{V}]_{1: N_{\mathrm{P}}}^{\dagger},
$$

and the near optimal RF precoder is written as

$$
\mathbf{F}_{\mathrm{RF}}^{\star}=\exp \left(j \angle\left([\mathbf{V}]_{1: N_{\mathrm{P}}} \mathbf{A}^{\star}\right)\right) .
$$

To this end, with the perfect CSIT, the hybrid precoding design problem is solved. In the next section, we will discuss the codebook-based hybrid precoder design without CSIT.

\section{Codebook Based Minimum Subspace DistorTION HYBRID PRECODING}

In this section, we assume that the CSIT is not available but the receiver obtains the perfect channel knowledge using the pilots in the transmitted signals. We exploit the RF codebook to optimize the RF precoder at the receiver side and return the index of codewords using limited feedback channel.

In the scenario of hybrid precoding, pilots are precoded using the RF precoder at the transmitter. The

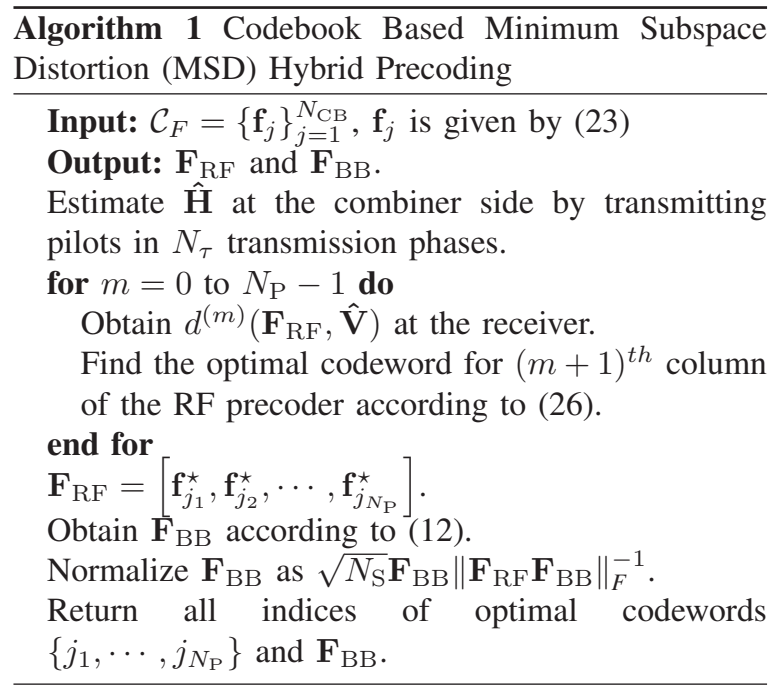

pilot symbols at the baseband of the transmitter are given by

$$
\mathbf{P}=\left[\mathbf{P}_{1}, \cdots, \mathbf{P}_{N_{\tau}}\right]
$$

where $\mathbf{P}_{j}$ is an $N_{\mathrm{P}} \times N_{\mathrm{P}}$ pilot symbol in the $j^{t h}$ transmission phase, and $N_{\tau}$ is the number of pilot transmissions. Since the RF precoder has $N_{\mathrm{P}}<N_{\mathrm{T}}$ columns, using a fixed RF precoder during the pilot transmission will lead to under-determined equations and hence $\hat{\mathbf{H}}$ cannot be estimated. Therefore, we use different RF precoder $\mathbf{F}_{\mathrm{RF}_{\mathrm{j}}}$ during different transmission phases. The detailed design of the pilots and precoders are illustrated in [14]. The received signal is given by

$$
\begin{aligned}
\mathbf{Y}= & \mathbf{H}\left[\mathbf{F}_{\mathrm{RF}_{1}}, \mathbf{F}_{\mathrm{RF}_{2}}, \cdots, \mathbf{F}_{\mathrm{RF}_{N_{\tau}}}\right] \times \\
& \operatorname{diag}\left(\left[\mathbf{P}_{1}, \mathbf{P}_{2}, \cdots, \mathbf{P}_{N_{\tau}}\right]\right)+\mathbf{Z},
\end{aligned}
$$

where $\mathbf{Z}$ is the noise matrix at the receiver. Using [15, eq. (6)] and noting that the mean value of the mmWave channel matrix is zero, we adopt the MMSE estimator of channel which is expressed as $\hat{\mathbf{H}}$.

Obtaining the estimated channel knowledge at the receiver, we adopt the codebook-based RF precoding method to provide near-optimal solutions to the optimization problem in (14). Due to the fact that the columns of the optimal hybrid precoder can be written as a linear combination of the array response vectors [4], we adopt array response vectors as codewords that are written as

$$
\begin{gathered}
\mathbf{f}_{j}=\exp \left[i \frac{2 \pi j}{N_{\mathrm{CB}}}\left(0,1, \cdots, N_{\mathrm{Tx}}-1\right)\right]^{T}, \\
j \in\left\{1,2, \cdots, N_{\mathrm{CB}}\right\} .
\end{gathered}
$$

Define the temporary RF precoder with the first $m$ codewords optimized as

$$
\begin{aligned}
\mathbf{F}_{\mathrm{RF}}^{(m)} & =\left[\mathbf{f}_{j_{1}}, \cdots, \mathbf{f}_{j_{m}}\right], \\
m & \in\left\{0, \cdots, N_{\mathrm{P}}\right\}, j \in\left\{1, \cdots, N_{\mathrm{CB}}\right\},
\end{aligned}
$$


where $\mathbf{f}_{j_{k}}, k \in\{1, \cdots, m\}$ are the optimized columns of the RF precoder.

We substitute $\mathbf{F}_{\mathrm{RF}}^{(m)}$ into (13) and introduce the concept of the subspace distortion as

$$
d^{(m)}\left(\mathbf{F}_{\mathrm{RF}}, \hat{\mathbf{V}}\right)=\left\|\left(\mathbf{F}_{\mathrm{RF}}^{(m)} \mathbf{F}_{\mathrm{RF}}^{(m)^{\dagger}}-\mathbf{I}_{N_{\mathrm{Tx}}}\right) \hat{\mathbf{V}}\right\|_{F},
$$

where $\hat{\mathbf{V}}$ is a estimated channel singular matrix given by the SVD of $\hat{\mathbf{H}}$, and $d^{(m)}\left(\mathbf{F}_{\mathrm{RF}}, \hat{\mathbf{V}}\right)$ is a fixed value as $\mathbf{F}_{\mathrm{RF}}^{(m)}$ is determined.

We optimize the RF precoder sequentially to reduce the calculation complexity. When $d^{(m)}\left(\mathbf{F}_{\mathrm{RF}}, \hat{\mathbf{V}}\right)$ is fixed, we design the $(m+1)^{\text {th }}$ column of RF precoder to further depress $d^{(m+1)}\left(\mathbf{F}_{\mathrm{RF}}, \hat{\mathbf{V}}\right)$. Therefore, the R$\mathrm{F}$ precoder optimization problem (14) is transformed into a joint codeword selection problem [9], which is expressed as

$$
\begin{aligned}
\mathbf{f}_{j}^{\star}=\arg \min & \left\|\left(\left[\mathbf{F}_{\mathrm{RF}}^{(m)}, \mathbf{f}_{j}\right]\left[\mathbf{F}_{\mathrm{RF}}^{(m)}, \mathbf{f}_{j}\right]^{\dagger}-\mathbf{I}_{N_{\mathrm{Tx}}}\right) \hat{\mathbf{V}}\right\|_{F} \\
\text { s.t. } \quad \mathbf{f}_{j} \in \mathcal{C}_{F}, & \forall j \in\left\{1, \cdots, N_{\mathrm{CB}}\right\} .
\end{aligned}
$$

The problem above can be solved via an exhaustive search of the codewords from the codebook. We iteratively increase $m$ from 0 to $N_{\mathrm{P}}-1$ to optimize and use a limited feedback channel to return all indices of the optimal codewords. The required number of the bits for the feedback is only $\log _{2}\left(N_{\mathrm{CB}}\right) N_{\mathrm{P}}$. This number of bits is much smaller compared with that in the solution to feedback the entire channel knowledge $\mathbf{H}$ or $\mathbf{V}$.

Once the RF precoding matrix is determined, the capacity of the combined digital channel, including the propagation channel and the RF precoder, is determined. There could be now various options for designing the digital precoder and combiner. Here, we adopt the solution proposed in (12) and (15) to determine the digital precoder and combiner. Notice that the dimension of $\mathbf{F}_{\mathrm{BB}}$ is rather smaller than that of $\mathbf{V}$, we can return the quantized norms and phases of each entries in $\mathbf{F}_{\mathrm{BB}}$ using the feedback channel.

\section{Simulation Results}

In this section, we present some simulation results to demonstrate the performance of the proposed MSD hybrid precoding design in a mmWave channel, and compare the achieved system performance with that for the unconstrained digital solution.

We adopt a mmWave channel model illustrated in Section II, where the channel has $L_{\mathrm{C}}=5$ clusters. The azimuth AoA and AoD are uniformly distributed in $\left[-180^{\circ}, 180^{\circ}\right]$, and the center elevation AoA and AoD are uniformly distributed in $\left[-90^{\circ}, 90^{\circ}\right]$ when a uniform planar array (UPA) is used in the simulation. Each cluster is contributed by $L_{\mathrm{R}}=6$ rays of which AoA and AoD are assumed to be Laplacian distributed with angular spread of $5^{\circ}$ [7]. Both UPA and ULA

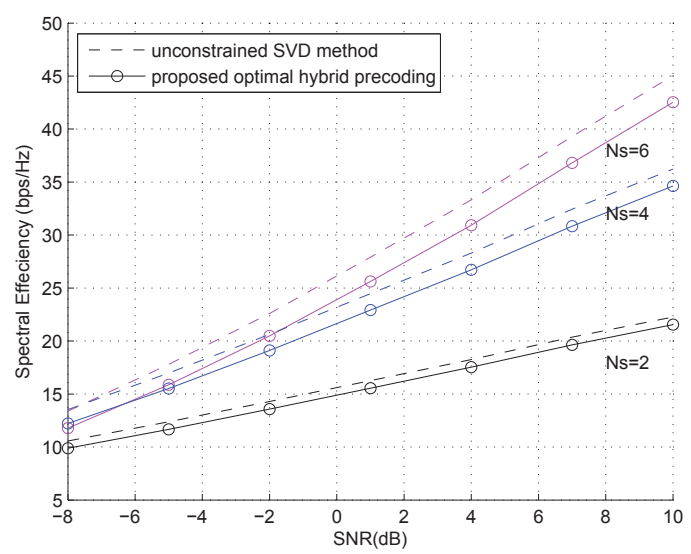

Fig. 2. Comparison between the unconstrained digital precoding and the proposed hybrid precoding with 2,4 or 6 data streams. The adopted system model has an $8 \times 8$ UPA at the transmitter and a $4 \times 4$ UPA at the receiver. Both transmitter and receiver adopt $N_{\mathrm{P}}=8 \mathrm{RF}$ chains.

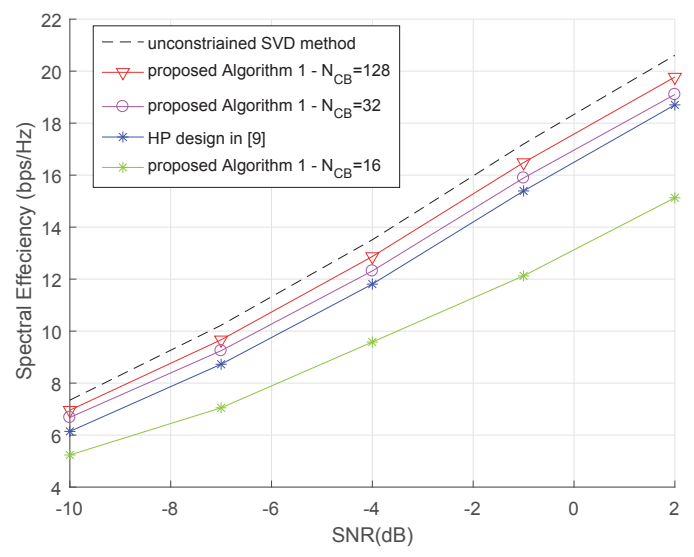

Fig. 3. Performance achieved by the proposed Algorithm 1 with a quantized codebook, where $N_{\mathrm{CB}}=16,32$ or 128 . The experimental setup is: a $32 \times 1$ ULA, $N_{\mathrm{P}}=8 \mathrm{RF}$ chains at the transmitter, an $8 \times 1 \mathrm{ULA}$ at the receiver, and $N_{\mathrm{S}}=4$ streams at both transmitter and receiver.

types are simulated, and the antenna spacing is half a wavelength. For simplicity, all clusters are assumed to be of equal power, i.e., $\alpha_{c, l}=\alpha_{c}$.

Fig. 2 presents the system performance in the case of perfect CSI. The numerical SE is calculated using (7). For the proposed system model, a single-user UPA system with $8 \times 8$ antennas at transmitter and $4 \times 4$ antennas at receiver is adopted. It is shown that the proposed optimal hybrid precoder approaches to the unconstrained precoding solution in terms of SE. With the number of data streams increasing, the SE increases but the increment rate of SE declines significantly. This is because the elements in the channel gain matrix decreases in this case. Adopting water-filling power control policy can potentially stop the declination of the increment rate. 


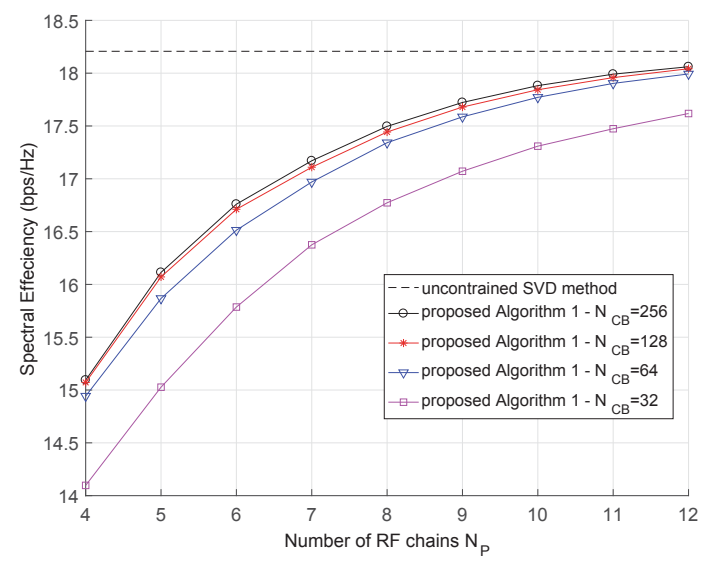

Fig. 4. Performance achieved by the proposed Algorithm 1 with SNR fixed at $0 \mathrm{~dB}$ and $N_{\mathrm{CB}}=32,64,128$ or 256 . The experimental setup is: a $32 \times 1$ ULA at the transmitter, an $8 \times 1$ ULA at the receiver, and $N_{\mathrm{S}}=4$ streams at both transmitter and receiver.

Fig. 3 shows the SE achieved by Algorithm 1 with a quantized codebook. A single-user system with a $32 \times 1$ ULA at transmitter and a $16 \times 1$ ULA at receiver is adopted. Both transmitter and receiver have $N_{\mathrm{P}}=8 \mathrm{RF}$ chains and $N_{\mathrm{S}}=4$ data streams. The tested codebook is quantized to 7 bits, 5 bits or 4 bits, and hence $N_{\mathrm{CB}}$ is 128,32 , or 16 accordingly. We compare the proposed algorithm to the algorithm proposed in [9] in the figure too. From the results, we notice that Algorithm 1 performs well with a 5 bit or larger quantized codebook and approaches to the unconstrained SVD method. The SE drops sharply when $N_{\mathrm{CB}}=16$, because when codebook is small, the subspace distortion does not reach its minimum point.

Fig. 4 illustrates how the system performance is influenced by the number of RF chains when Algorithm 1 is applied. The SNR is fixed to $0 \mathrm{~dB}$, and other set-up is the same as that in Fig. 3. With increasing the number of the RF chains, we can see that the spectral efficiency increases and the increment rate declines gradually. The $\mathrm{SE}$ is improved when increasing the codebook size from 32 to 128 , but there is almost no improvement after 128 . Hence, the subspace distortion between $\mathbf{F}_{\mathrm{RF}}$ and $\hat{\mathbf{V}}$ can be minimized using a relatively small codebook.

\section{CONCLUSIONS}

In this paper, we developed a minimum subspace distortion (MSD) hybrid precoding algorithm for the single-user mmWave system. Considering the mutual information maximization problem, we derived a near-optimal closed-form solution for a hybrid array in mmWave system. The proposed hybrid precoding design with prefect CSIT approaches the fully digital solution from the simulation results. Inspired by the developed closed-form solution, we adopted codebook based MSD hybrid precoding to remove the require- ment for CSIT. Simulation results show that the achievable spectral efficiency of the proposed hybrid precoding algorithm, with the use of a very small codebook, approaches to that of a fully digital solution. For future work, it would be valuable to extend the MSD hybrid precoding architecture to multi-user mmWave systems.

\section{REFERENCES}

[1] J. A. Zhang, X. Huang, V. Dyadyuk and Y. J. Guo, "Massive hybrid antenna array for millimeter-wave cellular communications," in IEEE Wireless Communications, vol. 22, no. 1, pp. 79-87, February 2015.

[2] R. Heath Jr, N. Gonzalez-Prelcic, S. Rangan, W. Roh, and A. Sayeed, "An overview of signal processing techniques for millimeter wave MIMO systems," IEEE J. Sel. Topics Signal Process., vol. 10, no. 4, pp. 436-453, Apr. 2016.

[3] T. S. Rappaport, R. W. Heath Jr., R. C. Daniels, and J. N. Murdock, Millimeter Wave Wireless Communications. Upper Saddle River, NJ, USA: Prentice-Hall, Sep. 2014.

[4] O. El Ayach, S. Rajagopal, S. Abu-Surra, Z. Pi, and R. Heath, "Spatially sparse precoding in millimeter wave MIMO systems," IEEE Trans. Wireless Commun., vol. 13, no. 3, pp. 1499-1513, Mar. 2014

[5] C. Kim, T. Kim, and J.-Y. Seol, "Multi-beam transmission diversity with hybrid beamforming for MIMO-OFDM systems," in IEEE Globecom Workshops. (GC Wkshps), pp. 61-65, Dec. 2013.

[6] L. Liang, W. Xu, and X. Dong,"Low-complexity hybrid precoding in massive multiuser MIMO systems," IEEE Commun. Lett., vol. 3, no. 6, pp. 653-656, Dec. 2014.

[7] C. Rusu, R.Rial, N.Prelcic, and R. Heath, "Low Complexity Hybrid Precoding Strategies for Millimeter Wave Communication Systems," IEEE Trans. Wireless Commun., vol.15, no. 12, pp. 8380-8393, Dec. 2016

[8] A. Alkhateeb, O. El Ayach, G. Leus, and R. W. Heath, Jr., "Channel estimation and hybrid precoding for millimeter wave cellular systems" IEEE J. Sel. Topics Signal Process., vol. 8, no. 5, pp. 831-846, Oct. 2014

[9] S. He, J. Wang, Y. Huang, B. Ottersten, and W. Hong, "Codebook-based hybrid precoding for millimeter wave multiuser systems," IEEE Transactions on Signal Processing, vol.65, no.20, pp. 5289-5304, Oct. 2017.

[10] Ahmed Alkhateeb, and R.Heath, "Frequency Selective Hybrid Precoding for Limited Feedback Millimeter Wave Systems," IEEE Trans. Wireless Commun., vol.64, no. 5, May. 2016.

[11] Ahmed Alkhateeb, G. Leus, and R. Heath, "Limited Feedback Hybrid Precoding for Multi-User Millimeter Wave Systems," IEEE Trans. Wireless Commun., vol. 14, no. 11, pp. 6491-6494, Nov. 2015.

[12] J. Singh and S. Ramakrishna, "On the feasibility of codebookbased beamforming in millimeter wave systems with multiple antenna arrays," IEEE Trans. Wireless Commun., vol. 14, no. 5, pp. 2670-2683, May 2015

[13] R. A. Pitaval and O. Tirkkonen, "Joint Grassmann-Stiefel quantization for MIMO product codebooks," IEEE Trans. Wireless Commun., vol. 13, no. 1, pp. 210-222, Jan. 2014.

[14] Nima N. Moghadam, H. S. Ghadikolaei, G. Fodor, M. Bengtsson, and Carlo Fischione, "Pilot Precoding and Combining in Multiuser MIMO Networks," IEEE Journal on Select Areas in Communications., vol. 35, no. 7, pp. 1632-1648, Jul. 2017.

[15] E. Bjornson and B. Ottersten, "A framework for training-based estimation in arbitrarily correlated Rician MIMO channels with Rician disturbance," IEEE Trans. Signal Process., vol. 58, no. 3, pp. 1807-1820, Mar. 2010. 